\title{
Economic Fourth Order Three-stage Method for Solving Systems of Second Order Differential Equations with Special Structure
}

\author{
Igor V. Olemskoy, Alexey S. Eremin*, Oksana S. Firyulina, and Aleksei A. Nechiporuk \\ St.-Petersburg State University, 7/9 Universitetskaya nab., St. Petersburg, 199034, Russia \\ *Email: a.eremin@spbu.ru
}

\begin{abstract}
An explicit embedded pair of methods for systems of second order ordinary equations with special structure is considered. Two-parametric families of methods of orders four and three with automatic step-size control are constructed. The numeric comparison to known embedded Runge-Kutta pairs of the same order is held.
\end{abstract}

\section{INTRODUCTION}

In [1] for systems of the form

$$
\begin{aligned}
& y_{i}^{\prime}=f_{i}\left(x, y_{1}, \ldots, y_{i-1}, y_{l+1}, \ldots, y_{n}\right), \quad i=\overline{1, l} \\
& y_{j}^{\prime}=f_{j}\left(x, y_{1}, \ldots, y_{j-1}\right), \quad j=\overline{l+1, n}, \quad n \geq 3
\end{aligned}
$$

an economic embedded explicit Runge-Kutta type method with automatic step-size control was constructed. The method is of order 4 with order 3 error estimation and has 4 stages with the first stage for the group (1) being the same as the last of the previous step. The method is denoted as RKS4(3)4F with the denotation $\operatorname{RKS} p(q) m \mathrm{~F}$ meaning Runge-Kutta type Structural method of order $p$ with order $q$ embedded estimator, with $m$ stages and FSAL.

It was modified so that the error estimation is made only for functions of the group (1). So only three stages are required for the group (2) and effectively the method has just three stages for all unknown functions. Such modification is denoted RKS4(3)(4,3)F

Both methods have shown their effectiveness compared to the known embedded Runge-Kutta pairs of order 4 and 3. In general, methods for systems with special structure analogous to (1)-(2) are called structural. Methods for most general structure are considered in [2]-[4].

Here methods for direct integration of systems of second order equations of the form

$$
\left\{\begin{array}{l}
x^{\prime \prime}=G\left(t, x, y, y^{\prime}\right), \\
y^{\prime \prime}=Q\left(t, x, y, x^{\prime}\right) .
\end{array}\right.
$$

are constructed on base of RKS4(3)4F and RKS4(3)(4,3)F. For example, the well-known restricted three-body problem is described with such system. The equations [5]

$$
\begin{aligned}
x_{1}^{\prime \prime} & =x_{1}+2 x_{2}^{\prime}-\mu^{\prime} \frac{x_{1}+\mu}{D_{1}}-\mu \frac{x_{1}-\mu^{\prime}}{D_{2}}, \\
x_{2}^{\prime \prime} & =x_{2}-2 x_{1}^{\prime}-\mu^{\prime} \frac{x_{2}}{D_{1}}-\mu \frac{x_{2}}{D_{2}}, \\
D_{1} & =\left(\left(x_{1}+\mu\right)^{2}+x_{2}^{2}\right)^{3 / 2}, \quad D_{2}=\left(\left(x_{1}-\mu^{\prime}\right)^{2}+x_{2}^{2}\right)^{3 / 2}, \\
\mu & =0.012277471, \quad \mu^{\prime}=1-\mu,
\end{aligned}
$$

rewritten with $y_{1}=x_{1}^{\prime}, y_{2}=x_{2}, y_{3}=x_{2}^{\prime}, y_{4}=x_{1}$ notations as a system of first-order equations, can be considered as a system (1)-(2) with $l=2, n=4$.

Since in the system (3) right-hand sides depend on firstderivatives special Runge-Kutta-Nyström methods (RKNs) for second-order equations are inapplicable, and direct application of standard Runge-Kutta methods cannot improve the stage to order ratio [6]. However it is possible to use structural methods for solving the system of first order equations derived from (3) and then rewrite them for direct application to the system of second order. This preserve the advantage of structural methods over classic Runge-Kutta methods.

\section{INTEGRATION METHOD}

To make the idea more clear we write down the method RKS4(3)(4,3)F for the system (3) in the full form. Assuming the values $x(t), x^{\prime}(t), y(t)$ and $y^{\prime}(t)$ known, the approximations $X, X^{\prime}, Y$ and $Y^{\prime}$ to $x(t+h), x^{\prime}(t+h), y(t+h)$ and $y^{\prime}(t+h)$ are found as

$$
\begin{aligned}
& X=x(t)+h x^{\prime}(t)+\frac{1}{10} h k_{1}^{G}+\frac{1}{3} h k_{2}^{G}+\frac{1}{15} h k_{3}^{G}, \\
& X^{\prime}=x^{\prime}(t)+\frac{1}{10} k_{1}^{G}+\frac{1}{2} k_{2}^{G}+\frac{2}{5} k_{3}^{G}, \\
& Y=y(t)+h y^{\prime}(t)+\frac{1}{3} h k_{1}^{Q}+\frac{1}{6} h k_{2}^{Q}, \\
& Y^{\prime}=y^{\prime}(t)+\frac{2}{5} k_{1}^{Q}+\frac{1}{2} k_{2}^{Q}+\frac{1}{10} k_{3}^{Q},
\end{aligned}
$$

where

$$
\begin{aligned}
k_{1}^{G}= & h G\left(t, x(t), y(t), y^{\prime}(t)\right), \\
k_{1}^{Q}= & h Q\left(t+\frac{1}{6} h, x(t)+\frac{1}{6} h x^{\prime}(t)+\frac{1}{36} h k_{1}^{G},\right. \\
& \left.y(t)+\frac{1}{6} h y^{\prime}(t), x^{\prime}(t)+\frac{1}{6} k_{1}^{G}\right), \\
k_{2}^{G}= & h G\left(t+\frac{1}{3} h, x(t)+\frac{1}{3} h x^{\prime}(t)+\frac{1}{18} h k_{1}^{G},\right. \\
& \left.y(t)+\frac{1}{3} h y^{\prime}(t)+\frac{1}{18} h k_{1}^{Q}, y^{\prime}(t)+\frac{1}{3} k_{1}^{Q}\right),
\end{aligned}
$$




$$
\begin{aligned}
k_{2}^{Q}= & h Q\left(t+\frac{2}{3} h, x(t)+\frac{2}{3} h x^{\prime}+\frac{5}{72} h k_{1}^{G}+\frac{1}{8} h k_{2}^{G},\right. \\
& \left.y(t)+\frac{2}{3} h y^{\prime}(t)+\frac{1}{4} h k_{1}^{Q}, x^{\prime}(t)-\frac{1}{12} k_{1}^{G}+\frac{3}{4} k_{2}^{G}\right), \\
k_{3}^{G}= & h G\left(t+\frac{5}{6} h, x(t)+\frac{5}{6} h x^{\prime}(t)+\frac{5}{144} h k_{1}^{G}+\frac{5}{16} h k_{2}^{G},\right. \\
& y(t)+\frac{5}{6} h y^{\prime}(t)+\frac{5}{18} h k_{1}^{Q}+\frac{5}{72} h k_{2}^{Q}, \\
& \left.y^{\prime}(t)+\frac{5}{12} k_{1}^{Q}+\frac{5}{12} k_{2}^{Q}\right), \\
k_{3}^{Q}= & h Q\left(t+h, x(t)+h x^{\prime}(t)-\frac{1}{24} h k_{1}^{G}+\frac{5}{8} h k_{2}^{G},\right. \\
& y(t)+h y^{\prime}(t)+\frac{5}{36} h k_{1}^{Q}+\frac{5}{18} h k_{2}^{Q}, \\
& \left.x^{\prime}(t)+\frac{3}{4} k_{1}^{G}-\frac{5}{12} k_{2}^{G}+\frac{2}{3} k_{3}^{G}\right) .
\end{aligned}
$$

In order to find the local error estimation the value

$$
k_{4}^{G}=h G\left(t+h, X, Y, Y^{\prime}\right)
$$

is found and

$$
\begin{gathered}
\bar{X}=x(t)+h x^{\prime}(t)+\left(\frac{1}{10}-\frac{2}{15} \eta\right) h k_{1}^{G}+\left(\frac{1}{3}+\frac{1}{6} \eta\right) h k_{2}^{G} \\
+\left(\frac{1}{15}+\frac{2}{15} \eta\right) h k_{3}^{G}-\frac{1}{6} \eta h k_{4}^{G}, \\
\bar{X}^{\prime}=x^{\prime}(t)+\left(\frac{1}{10}-\frac{2}{5} \xi\right) k_{1}^{G}+\left(\frac{1}{2}+\xi\right) k_{2}^{G} \\
+\left(\frac{2}{5}-\frac{8}{5} \xi\right) k_{3}^{G}+\xi k_{4}^{G}, \\
\bar{Y}=y(t)+h y^{\prime}(t)+\left(\frac{1}{3}+\frac{1}{15} \xi\right) h k_{1}^{Q} \\
+\left(\frac{1}{6}-\frac{1}{6} \xi\right) h k_{2}^{Q}+\frac{1}{10} \xi h k_{3}^{Q},
\end{gathered}
$$

So

$$
\begin{aligned}
x(t+h) & =X+O\left(h^{5}\right)=\bar{X}+O\left(h^{4}\right), \\
x^{\prime}(t+h) & =X^{\prime}+O\left(h^{5}\right)=\bar{X}^{\prime}+O\left(h^{4}\right), \\
y(t+h) & =Y+O\left(h^{5}\right)=\bar{Y}+O\left(h^{4}\right), \\
y^{\prime}(t+h) & =Y^{\prime}+O\left(h^{5}\right) .
\end{aligned}
$$

The parameters $\eta$ and $\xi$ are non-zero and can be chosen arbitrarily. Let's denote the method (5)-(9) RKNS4(3)(4,3)F (though in fact it isn't an RKN).

In order to estimate the local error for all variables we need the value

$$
\begin{aligned}
k_{4}^{Q}= & h Q\left(t+\frac{5}{6} h,\right. \\
& x(t)+\frac{5}{6} h x^{\prime}(t)-\frac{1}{12} h k_{1}^{G}+\frac{1}{4} h k_{2}^{G}+\frac{1}{36} k_{4}^{G}, \\
& y(t)+\frac{5}{6} h y^{\prime}(t)+\frac{4}{15} h k_{1}^{Q}+\frac{1}{12} h k_{2}^{Q}-\frac{1}{60} k_{3}^{Q}, \\
& \left.x^{\prime}(t)+\frac{1}{10} k_{1}^{G}+\frac{1}{2} k_{2}^{G}+\frac{2}{5} k_{3}^{G}-\frac{1}{6} k_{4}^{G}\right) .
\end{aligned}
$$

So it gives

$$
\begin{aligned}
\bar{Y}^{\prime}=y^{\prime}(t)+\left(\frac{2}{15}+\frac{1}{15} \eta\right) k_{1}^{Q} & +\left(\frac{1}{2}-\frac{2}{3} \eta\right) k_{2}^{Q} \\
+ & \left(\frac{1}{10}-\frac{2}{5} \eta\right) k_{3}^{Q}+\eta k_{4}^{Q},
\end{aligned}
$$

which is order 3 approximation to $y^{\prime}(t+h)$. Unfortunately $k_{4}^{Q}$ cannot be reused at the next step. But still, the method (now denoted RKNS4(3)4F) is more effective than known methods.

\section{TEST COMPUTATIONS}

The methods were tested and compared on two problems.

Test 1. The methods RKNS4(3)4F and RKNS4(3)(4,3)F are compared to the classic four-stages RungeKutta method ("The" RungeKutta method) with five-stages third-order estimator, denoted RK4(3)T in [7], and to the same RungeKutta method but with an embedded second-order estimator (named

\begin{tabular}{|c|c|c|c|c|c|c|c|c|}
\hline \multirow[t]{2}{*}{$\log _{10} \| \delta \mid$} & \multicolumn{2}{|c|}{ RKNS4(3)4F } & \multicolumn{2}{|c|}{ RKNS4(3)(4,3)F } & \multicolumn{2}{|c|}{ RK4(3)T } & \multicolumn{2}{|c|}{ RK4(2) } \\
\hline & $N_{h}$ & $N_{G+Q}$ & $N_{h}$ & $N_{G+Q}$ & $N_{h}$ & $N_{G+Q}$ & $N_{h}$ & $N_{G+Q}$ \\
\hline-6.00 & 156 & 1092 & 161 & 966 & 234 & 1872 & 236 & 1888 \\
\hline-6.50 & 211 & 1477 & 218 & 1308 & 310 & 2480 & 312 & 2480 \\
\hline-7.00 & 279 & 1953 & 292 & 1752 & 413 & 3304 & 417 & 3386 \\
\hline-7.50 & 377 & 2639 & 395 & 2370 & 553 & 4424 & 556 & 4444 \\
\hline-8.00 & 496 & 3472 & 524 & 3144 & 734 & 5872 & 738 & 5904 \\
\hline-8.50 & 661 & 4627 & 708 & 4248 & 978 & 7824 & 983 & 7864 \\
\hline-9.00 & 881 & 6167 & 955 & 5730 & 1304 & 10432 & 1311 & 10448 \\
\hline-9.50 & 1174 & 8218 & 1280 & 7680 & 1737 & 13896 & 1747 & 13976 \\
\hline-10.00 & 1567 & 10969 & 1740 & 10440 & 2321 & 18568 & 2332 & 18656 \\
\hline-10.50 & 2087 & 14609 & 2325 & 13950 & 3089 & 24712 & 3106 & 24848 \\
\hline-11.00 & 2783 & 19481 & 3110 & 18660 & 41 & 32928 & 4141 & 33128 \\
\hline-11.50 & 3712 & 25984 & 4172 & 25032 & 5490 & 43920 & 5522 & 44176 \\
\hline
\end{tabular}
in Russian mathematical tradition "Egorov control term" after Prof. Vsevolod A. Egorov [8] denoted RK4(2). They were used to solve the problem

$$
\begin{aligned}
x^{\prime \prime} & =-2 x+\frac{1}{2} y^{\prime}, \quad y^{\prime \prime}=-\frac{1}{2} x^{\prime}-2 y, \\
x(0) & =x^{\prime}(0)=1, \quad y(0)=2, \quad y^{\prime}(0)=3,
\end{aligned}
$$

in the interval $t \in[0,2 \pi]$. The general solution of (12) is

$$
\begin{gathered}
x(t)=C_{1} \cos (\alpha t)+C_{2} \sin (\alpha t)+C_{3} \cos (\beta t)+C_{4} \sin (\beta t), \\
y(t)=-C_{1} \sin (\alpha t)+C_{2} \cos (\alpha t)-C_{3} \sin (\beta t)+C_{4} \cos (\beta t), \\
\alpha=\frac{1-\sqrt{33}}{4}, \quad \beta=\frac{1+\sqrt{33}}{4} .
\end{gathered}
$$

The effectiveness of any method depends on the algorithm of step-size control, and all the methods RKNS4(3)4F, RKNS4(3)(4,3)F and RK4(3)T, RK4(2) are of the same class of one-step methods. So all of them were realised with the similar algorithmic core. The new step-size was calculated as $h_{\text {new }}=0.9 \cdot h(t o l / e r r)^{1 /(q+1)}$, where tol is the local error tolerance and err is the measured local error.

The problem was solved with different tolerance values so that the global error $\delta$ changed from $10^{-6}$ to $10^{-11.5}$. All four methods showed the expected convergence, but it should be noted that the step-size control was more sensitive in case of RKNS4(3)4F and RKNS4(3)(4,3)F.

In the Table I the number of steps $N_{h}$ and the number of right-hand side calculations $N_{G+Q}$ over the whole interval $[0,2 \pi]$ which provide the norm of global error $\|\delta\|$ are given.

TABLE I

COMPARISON FOR THE TEST PROBLEM 1.

The results confirm theoretical expectations. The global error to right-hand side computations ratio show that for the 


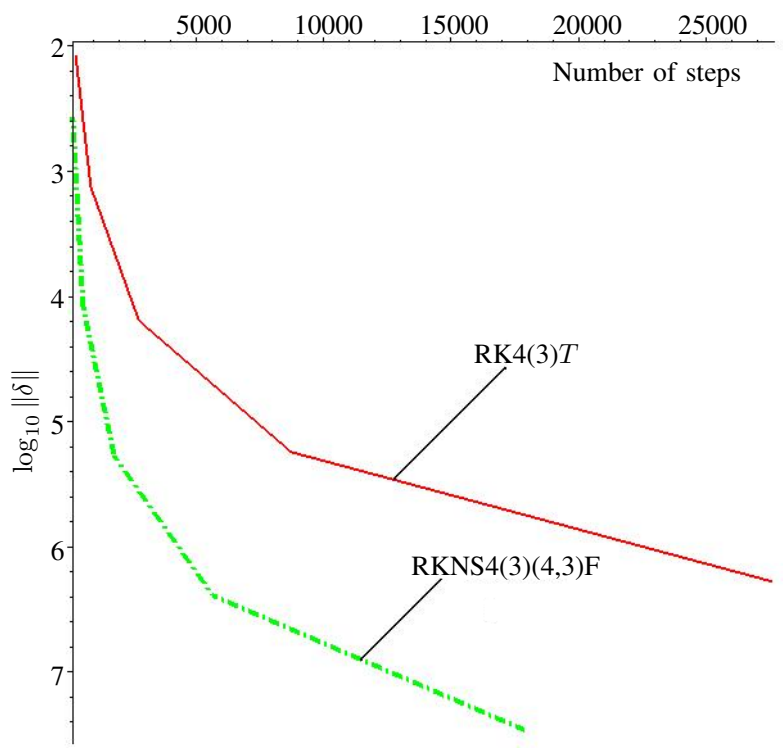

Fig. 1. Global error to number of steps ratio comparison in Test 2.

same $\|\delta\|$ values RKNS4(3)4F and RKNS4(3)(4,3)F demand fewer steps and right-hand side evaluations. And for the same computational cost RK4(3)T and RK4(2) are less effective than RKNS4(3)4F and RKNS4(3)(4,3)F in sense of global error value.

Test 2. The restricted three-body problem (4) with specially chosen initial values, for example

$$
\begin{aligned}
x_{1}(0) & =0.994, \quad x_{1}^{\prime}(0)=0, \quad x_{2}(0)=0, \\
x_{2}^{\prime}(0) & =2.00158510637908252240537862224, \\
t_{\text {end }} & =17.0652165601579625588917206249,
\end{aligned}
$$

has periodic solution with period $t_{\text {end }}$ [6]. Such orbits are often called "Arenstorf orbits". The initial value problem was solved up to $t_{\text {end }}$ with RKNS4(3)(4,3)F with $\xi=\eta=3$ (in form (3)) and with RK4(3)T (in form (1)-(2)) and the deviation in the end from the initial value was measured, being used as a global error $\delta$ evaluation.
In the Figure 1 the global error to the number of steps ratio for both methods are presented. RKNS4(3)(4,3)F provides the same accuracy with fewer steps, and moreover takes fewer stages per step. This confirms, as it was expected by the construction, its better performance in comparison with classic Runge-Kutta schemes.

\section{CONCLUSION}

The numeric tests confirm that methods exploiting special structure of ODE systems are more effective. Specially constructing them with different computational schemes for different parts of systems one can obtain the demanded convergence order and global error with fewer stages than with usual Runge-Kutta methods.

Direct implementation of such methods to systems of second-order equations keeps the mentioned effectiveness. Their field of application is wider than of known RungeKutta-Nyström methods.

\section{REFERENCES}

[1] I. V. Olemskoy, A. S. Eremin, "An Embedded Fourth Order Method for Solving Structurally Partitioned Systems of Ordinary Differential Equations," Appl. Math. Sci., vol. 9, pp. 4843-4852, 2015.

[2] I. V. Olemskoy, "Fifth-order four-stage method for numerical integration of special systems," Comp. Math. Math. Phys., vol. 42, no. 8, pp. 1135$1145,2002$.

[3] I. V. Olemskoy, "Structural approach to the design of explicit one-stage methods," Comp. Math. Math. Phys., vol. 43, no. 7, pp. 961-974, 2003.

[4] I. V.Olemskoy, "The modification of the algorithm for determining structural peculiarities," (In Russian), Vestn. St.-Petersburg U., ser. 10, no. 2, pp. 46-54, 2006.

[5] V. Szebehely, Theory of orbits. The restricted problem of three bodies. New York: Acad. Press, 1967.

[6] E. Hairer, S. P. Nørsett, G. Wanner, Solving Ordinary Differential Equations I: Nonstiff Problems, 3rd ed. Springer-Verlag Berlin Heidelberg, 2008.

[7] J. R. Dormand, P. J. Prince, "New Runge-Kutta algorithms for numerical simulation in dynamical astronomy," Celestial Mech., vol. 18, pp. $223-$ 232, 1978

[8] O. B. Arushanyan, S. F.Zaletkin, Numerical Solution of Ordinary Diffential Equations with Fortran, (In Russian), Moscow: Moscow State University, 1990. 